\title{
Cerebellar liponeurocytoma: a rare intracranial tumor with possible familial predisposition. Case report
}

\author{
Amparo Wolf, MD, PhD, ${ }^{1}$ Huda Alghefari, MD, ${ }^{2}$ Daria Krivosheya, MD, MSc, ${ }^{1}$ \\ Michael D. Staudt, MD, MSc, ${ }^{1}$ Gregory Bowden, MD, ${ }^{1}$ David R. Macdonald, MD, ${ }^{1,3}$ \\ Sharan Goobie, MD, MSc, ${ }^{4}$ David Ramsay, MB, ChB, DPhil, ${ }^{2}$ and Matthew O. Hebb, MD, PhD'
}

Departments of ${ }^{1} \mathrm{Clinical}$ Neurological Sciences, ${ }^{2}$ Pathology, ${ }^{3}$ Oncology, and ${ }^{4}$ Pediatrics, London Health Sciences Centre, London Regional Cancer Centre, and Western University Schulich School of Medicine and Dentistry, London, Ontario, Canada

\begin{abstract}
The biological origin of cerebellar liponeurocytomas is unknown, and hereditary forms of this disease have not been described. Here, the authors present clinical and histopathological findings of a young patient with a cerebellar liponeurocytoma who had multiple immediate family members who harbored similar intracranial tumors. A 37-year-old otherwise healthy woman presented with a history of progressive headaches. Lipomatous medulloblastoma had been diagnosed previously in her mother and maternal grandfather, and her maternal uncle had a supratentorial liponeurocytoma. MRI revealed a large, poorly enhancing, lipomatous mass emanating from the superior vermis that produced marked compression of posterior fossa structures. An uncomplicated supracerebellar infratentorial approach was used to resect the lesion. Genetic and histopathological analyses of the lesion revealed neuronal, glial, and lipomatous differentiation and confirmed the diagnosis of cerebellar liponeurocytoma. A comparison of the tumors resected from the patient and, 22 years previously, her mother revealed similar features. Cerebellar liponeurocytoma is a poorly understood entity. This report provides novel evidence of an inheritable predisposition for tumor development. Accurate diagnosis and reporting of clinical outcomes and associated genetic and histopathological changes are necessary for guiding prognosis and developing recommendations for patient care.
\end{abstract}

http://thejns.org/doi/abs/10.3171/2015.6.JNS142965

KEY WORDS lipomatous medulloblastoma; neurolipocytoma; brain tumor; familial; oncology

$\mathrm{C}$ EREBELLAR liponeurocytomas are typically lowgrade tumors that arise in the cerebellar hemispheres or vermis and contain cells of neuronal, astrocytic, and lipomatous differentiation. ${ }^{17}$ The biology and long-term outcomes of these tumors are undefined, and to our knowledge, familial occurrences have not been described. We present here the case of a young woman with a cerebellar liponeurocytoma and multiple immediate family members, including her mother, with similar lesions. This report describes the tumors from the patient and her mother and provides a brief overview of the histopathology and genetic changes associated with this disease.

\section{Case Report}

\section{Clinical Presentation}

A 37-year-old woman presented with a 2-year history of worsening morning and daytime headaches in the occipital area that radiated over the cranium. She also described intermittent paresthesia in her hands and feet, which was of variable distribution and duration. The patient was otherwise healthy with a grossly normal neurological examination. She smoked cigarettes but had no other known medical problems. CT and MRI of the head and spine revealed a solitary fat-containing tumor arising from the region of the superior cerebellar hemisphere and vermis. The heterogeneous lesion had numerous ill-defined septations with patchy enhancement and measured $4.4 \times 4.1 \times$ $4.3 \mathrm{~cm}$ in the anterior-posterior, medial-lateral, and superior-inferior dimensions, respectively. It extended through the incisura and involved the tectal plate and several deep veins, including the left basal vein of Rosenthal. There was complete effacement of the fourth ventricle and compensated dilation of the third and lateral ventricles. There was displacement of the cerebellar tonsils through the foramen magnum, with compression of the cervicomedullary junction (Fig. 1A and B).

ABBREVIATIONS GFAP = glial fibrillary acidic protein; IDH1 = isocitrate dehydrogenase 1; MAP-2 = microtubule-associated protein 2; MIB-1 = monoclonal antihuman Ki 67 . SUBMITTED December 30, 2014. ACCEPTED June 17, 2015.

INCLUDE WHEN CITING Published online November 27, 2015; DOI: 10.3171/2015.6.JNS142965. 


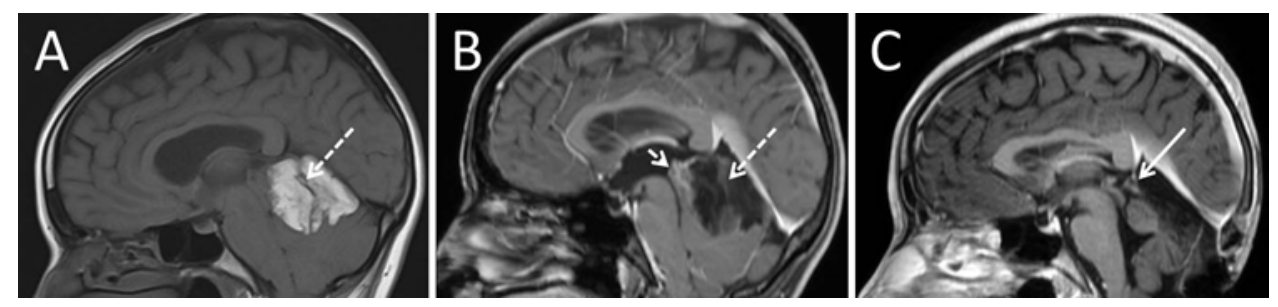

FIG. 1. Preoperative and postoperative neurological imaging of a cerebellar liponeurocytoma in the current patient. A: Sagittal T1-weighted Gd-enhanced MR image shows the large hyperintense lesion arising from the superior cerebellum, producing marked compression of the fourth ventricle, tectum, and cerebellum. B: Sagittal T1-weighted MR image with Gd and fat suppression. With this technique, the tumor mass is rendered hypointense, consistent with a significant lipomatous component, and the involved vasculature is more readily discerned. The tumor was only faintly enhancing, most prominently within the numerous septations (dashed arrows in A and B) and at the ventral aspect of the tumor and tectal plate (small arrow in B). C: Postoperative sagittal T1weighted MR image with Gd but no fat suppression. Near-total resection was achieved with a small remnant of tumor left adherent to deep veins near the galenic confluence (solid arrow).

\section{Family History}

Lipomatous medulloblastoma had been diagnosed in the patient's maternal grandfather decades earlier, and he reportedly died as a result of his disease. A maternal uncle had undergone resection of a supratentorial liponeurocytoma and remained alive at the time of this writing. The details of tumor histopathology are not available for these patients. In 1992, at 40 years of age, the patient's mother underwent resection of a posterior fossa tumor at our institution. The tumor was diagnosed as a "lipomatous medulloblastoma" (an entity now classified as a liponeurocytoma), and she subsequently received craniospinal radiation but no chemotherapy. This person is currently alive and free of active disease. The index patient has a younger sister and an 11-year-old son, both of whom are well but have not been formally screened for CNS tumors.

\section{Operation and Postoperative Course}

A wide midline suboccipital craniectomy, C-1 laminectomy, and duraplasty were performed to decompress the posterior fossa. Before tumor resection, an external ventricular drain was stereotactically placed through an occipital bur hole. The release of CSF, mild hyperventilation, and mannitol helped reduce intracranial pressure. Tumor resection was performed via a supracerebellar infratentorial approach. The numerous large cerebellar draining veins adherent to the tumor capsule were preserved. The lesion was extensive and relatively soft, but the dense septa made it tenacious, necessitating use of an ultrasonic aspirator for resection. There were many small arteries that fed the lesion, particularly in the ventral part of the mass. The tumor capsule was dissected from the deep venous system, which revealed a thickened veil of arachnoid over the vein of Galen confluence and the quadrigeminal cistern. At most surfaces, the tumor-brain interface could be developed; however, at the ventral vermis and tectum, this plane became indistinct. The left basal vein of Rosenthal coursed within a dense arachnoid/tumor complex. A small part of the neoplasm was left in situ to protect the involved deep veins and tectum (Fig. 1C). The patient remained clinically stable throughout her postoperative course and experienced no new neurological deficits.

\section{Tumor Histopathology and Cytogenetics}

The patient's tumor was a moderately cellular neoplasm formed by bland neoplastic neurons, oligodendroglial-like cells, and adipocytes. Neural rosettes were numerous. There was minimal nuclear pleomorphism, and there was no evidence of necrosis or tumor-related vascular changes. Adipocytes lay in and surrounded the cellular regions. The neurocytic cells were immunopositive for various neuronal and related markers, including NeuN, microtubuleassociated protein 2 (MAP-2), and synaptophysin. Glial fibrillary acidic protein (GFAP) immunolabeling was very focal and largely restricted to cell processes around the lipid vacuoles. The lesion was immunonegative for isocitrate dehydrogenase 1 (IDH1)-R132H. Rare nuclei were p53 immunopositive. No mitoses were observed, and less than $1 \%$ of the nuclei were monoclonal antihuman Ki 67 (MIB-1) immunopositive. There was patchy perivascular lymphocytosis scattered through the lesion. Fluorescence in situ hybridization showed no deletions of $1 \mathrm{p}, 19 \mathrm{q}$, or TP53 and no amplification of the epidermal growth factor receptor gene (Fig. 2).

In comparison, the mother's tumor was a moderately to densely cellular neoplasm that was well circumscribed and lightly calcified. There were focal areas of vascular hyalinosis without vascular proliferation. The tumor cells were predominantly spindle shaped with long eosinophilic processes. Oligodendroglial-like cells with perinuclear halos were seen in several areas. The adipocyte-like component was less prominent than in the daughter's tumor but was nevertheless well established. The immunoprofile was similar to that of the daughter's tumor, but there was no staining for p53. Contemporary fluorescence in situ hybridization analysis was not carried out because of severe degradation of the specimen (Fig. 3). Although the mother's tumor was classified as a lipomatous medulloblastoma at the time (1992), it conformed to the entity currently known as cerebellar liponeurocytoma.

\section{Discussion}

The first case of cerebellar liponeurocytoma, in a 44-year-old man, was described in 1978 by Bechtel et al. ${ }^{6}$ There are now more than 40 reports of these tumors 

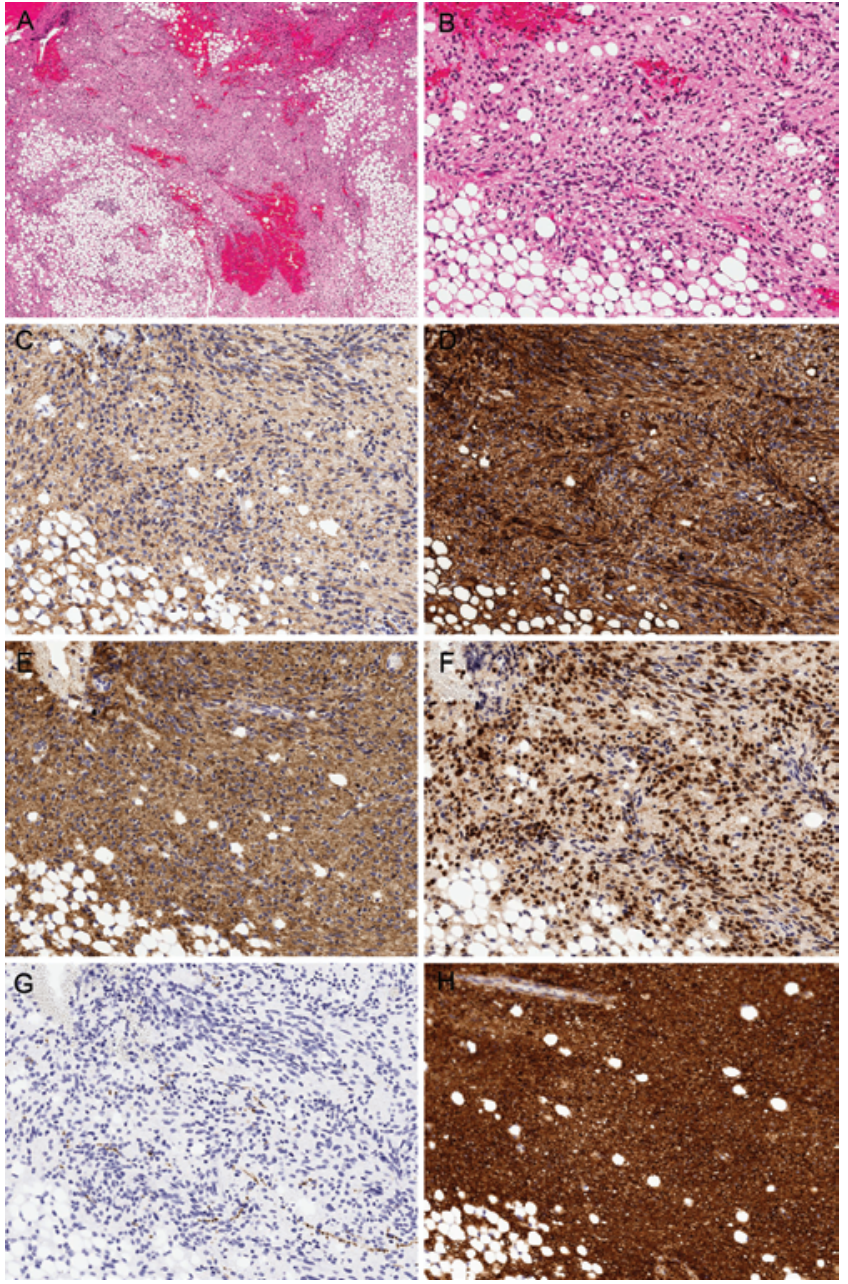

FIG. 2. Histological appearance of the patient's cerebellar liponeurocytoma. A: Neurocytic and lipomatous components are evident within the lesion. H \& E. B: The tumor consisted of a moderately cellular lesion with a neuropil-like background admixed with diffuse adipocyte-like tissue. Within the cellular zones, the cells had round-to-elongated stippled nuclei with mild nuclear polymorphism and minimal lightly eosinophilic cytoplasm. Rosettes were frequent. H \& E. C: Immunolabeling of the intermediate filament protein GFAP, indicating astrocytic differentiation, was focal, with prominence around vacuoles in lipidized tumor cells. D: The intermediate filament protein, vimentin, stained strongly and diffusely. The tumor expressed varying levels of the neuronal markers synaptophy$\sin (E)$, NeuN (F), neurofilament (G), and MAP-2 (H). Original magnification $\times 5(\mathrm{~A})$ and $\times 20(\mathrm{~B}-\mathrm{H})$. Panels $\mathrm{C}-\mathrm{H}$ show the respective antigens visualized with the chromogen, diaminobenzidine, and the sections counterstained with hematoxylin. Figure is available in color online only.

published under different diagnostic terms, including lipomatous or lipidized medulloblastoma, ${ }^{9}$ neurolipocytoma, ${ }^{10}$ medullocytoma,${ }^{11}$ lipomatous glioneurocytoma,${ }^{4}$ lipidized mature neuroectodermal tumor, ${ }^{12}$ and cerebellar liponeurocytoma. ${ }^{3,8,14,19}$ Cerebellar liponeurocytoma was introduced as a mixed neuronal-glial tumor in the $2000 \mathrm{WHO}$ classification of tumors of the CNS, with a Grade I designation to reflect the advanced neurocytic differentiation and favorable prognosis. ${ }^{16}$ Subsequent reports of tumor recurrence with atypical features prompted redesignation as
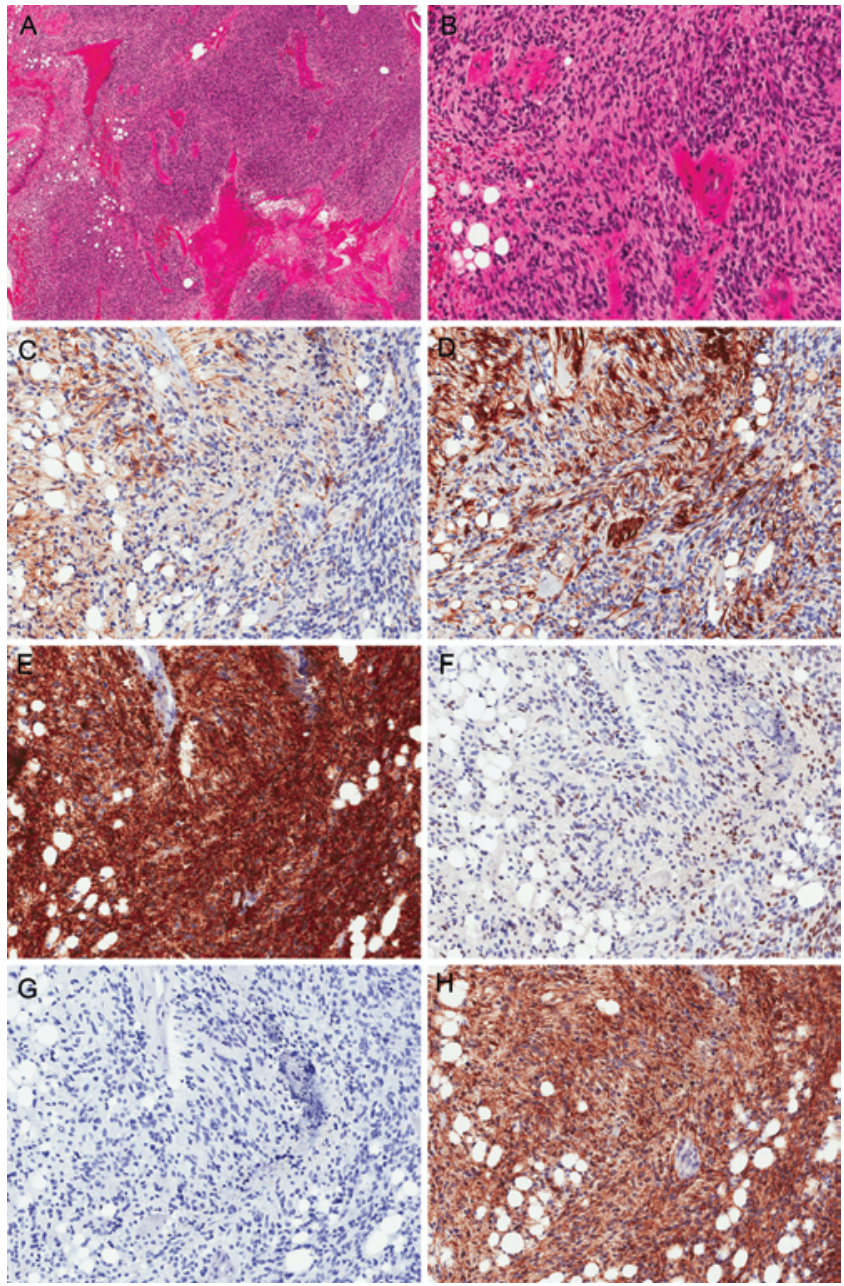

FIG. 3. Histological appearance of the mother's tumor. A and B: Neurocytic and lipomatous tumor fractions were evident. The neurocytic component was more pronounced than that in the daughter's tumor. $\mathrm{H}$ \& $\mathrm{E}$. There was focal, patchy staining of the intermediate filament proteins GFAP (C) and vimentin (D). The tumor stained strongly for the neuronal marker synaptophysin (E), but there was minimal immunolabeling for the more differentiated neuronal markers NeuN (F) and neurofilament (G). H: There was strong expression of the neuronal marker MAP-2. Original magnification $\times 5(\mathrm{~A})$ and $\times 20(\mathrm{~B}-\mathrm{H})$. Panels $\mathrm{C}-\mathrm{H}$ show the respective antigens visualized with the chromogen, diaminobenzidine, and the sections counterstained with hematoxylin. Figure is available in color online only.

Grade II in the 2007 revised WHO classification. ${ }^{13,17}$ These lesions contain a small-cell component accompanied by lipidized neoplastic cells that resemble mature adipocytes. Areas of neuronal differentiation are positive for markers such as synaptophysin and MAP-2. ${ }^{8}$ Reactivity for GFAP is usually present but focal. Fat-containing cells also typically express neuronal markers and GFAP, consistent with aberrant differentiation of tumor cells rather than them being true adipocytes. There is usually no histological evidence of anaplasia or rapid growth, such as mitosis, necrosis, or vascular hyperplasia. The Ki 67/MIB-1 index is usually low $(<5 \%)$; however, patients with a relatively high proliferation rate and cytopathologic atypia have been re- 
ported to have a greater risk of recurrence and, therefore, a poorer prognosis. ${ }^{2,7,14,20}$ There is currently no established standard of care, but maximal safe resection followed by postoperative imaging surveillance for recurrence seems prudent. The roles of adjuvant chemotherapy and radiation have not been established. ${ }^{18}$ In the current cases, lesions from the patient and her mother had comparable immunohistochemical profiles, including variable expression of synaptophysin, MAP-2, NeuN, neurofilament, GFAP, and vimentin. The patient's tumor appeared more differentiated, with a greater lipidized component and stronger expression of neuronal markers. There was minimal expression of $\mathrm{p} 53$ in both tumors and no evidence of the IDHI-R132H mutation, which is a favorable prognosticator associated with diffusely infiltrating gliomas.,22

The genetic and cellular origins of liponeurocytomas have not been defined. These rare tumors are usually found in the cerebellum, although supratentorial lesions have been described and seem to share common immunohistological profiles and neoplastic behavior. ${ }^{8,15,21}$ Central neurocytomas exhibit similar cellular attributes but have no lipomatous fraction. The presence of fat, typically detected as a hypodense mass on CT images or as a hyperintense mass on T1-weighted MR images (which converts to hypointense on fat-suppression T1-weighted images), helps distinguish liponeurocytoma from other tumor types. Although classic medulloblastomas may present occasionally with foamy histiocytes admixed with primitive neuroectodermal cells, the presence of typical nodular architecture, absence of vacuolated (adipocyte-like) tumor cells, and a high proliferative fraction $(15 \%-40 \%)$ differentiate these tumors from liponeurocytomas. ${ }^{13,17}$ In a study of 20 cerebellar liponeurocytomas, gene expression profiles and DNA sequencing results were compared with those of medulloblastomas and central neurocytomas..$^{13}$ The investigators reported a TP53 mutation frequency (20\%) in liponeurocytomas higher than that typically seen in either of the other tumors. No mutations in PTCH, APC, or $\beta$-catenin genes were identified in the cerebellar liponeurocytomas, each of which has been associated with a subset of medulloblastomas. Furthermore, isochromosome 17q, a genetic hallmark present in $40 \%$ of classic medulloblastomas, was not observed in any of the cerebellar liponeurocytomas. cDNA analysis generally related cerebellar liponeurocytomas to central neurocytomas, but this relation was tempered by TP53 mutations, which are absent in the latter and may reflect divergent genetic origins. Enhanced levels of the adipocyte-associated transcription factor, NEUROG1, and fatty acid-binding protein 4 have also been described in cerebellar liponeurocytomas. ${ }^{5}$ Given the obvious discrepancies in age of onset, tumor behavior, and associated genetic changes, it is likely that the majority, if not all, of the previously reported lipomatous medulloblastomas represent liponeurocytomas, and the use of the former term was discouraged in the revised WHO classification. ${ }^{17}$ Ependymoma cells and neoplastic oligodendrocytes can also resemble the small-cell component of liponeurocytoma. The prognostic implications and role of adjuvant chemoradiation in these other tumor types make their distinction from liponeurocytoma critical for optimized patient care.
There likely exists in this family a yet-to-be-identified germline mutation that predisposes to the formation of liponeurocytomas, with no other apparent tumor predisposition. The genetic underpinnings of tumor formation for this family are unknown; however, based on the available history, an autosomal dominant mode of inheritance is suspected. Several investigations would be feasible for helping to elucidate the genetic etiology. As an initial step, routine peripheral blood karyotyping could be performed to rule out a chromosomal translocation that may affect a known oncogene. Second, TP53 sequencing and deletion/ duplication testing on blood of affected family members would rule out a germline mutation, although it would be unusual for a germline TP53 mutation to result in only 1 isolated tumor type in multiple members of a family. Chromosomal microarray testing would rule out a small deletion or duplication along the chromosomes that could potentially involve a cancer-predisposition gene. Finally, whole-exome sequencing or whole-genome sequencing using next-generation sequencing technology would be a large-scale approach to screening for a germline mutation shared by the index patient and her mother. Ideally, one would find a germline mutation in a gene with a known role in oncogenesis; however, the use of this technology raises the possibility of finding hundreds to thousands of sequence variants of uncertain significance in genes with functions that may not be well understood. Particular attention should be given to genes involved in neurogenesis, because previous studies showed transcription factors, such as NEUROG1 and fatty acid-binding protein 4, to be enhanced in liponeurocytoma tissue. Available pathology samples may be useful for further investigation and functional studies of candidate genes. Determination of the underlying genetic defect in this family would enable genetic counseling, presymptomatic testing, and development of a screening protocol for at-risk individuals. Recommendations for CNS tumor screening of family members have not been established yet. Understanding the molecular pathology of such a mutation could also provide family members with information on whether they are at an increased risk for any other types of tumors.

Cerebellar liponeurocytomas are rare intracranial tumors without a known etiology. The family described herein is remarkable in that multiple individuals in several generations have been afflicted with this disease. To our knowledge, this is the first report of such a familial presentation, which is indicative of a hereditary origin. Additional studies are needed to define the causal genetic mutation(s), prognosis and treatment guidelines for cerebellar liponeurocytomas.

\section{References}

1. Agnihotri S, Aldape KD, Zadeh G: Isocitrate dehydrogenase status and molecular subclasses of glioma and glioblastoma. Neurosurg Focus 37(6):E13, 2014

2. Aker FV, Ozkara S, Eren P, Peker O, Armağan S, Hakan T: Cerebellar liponeurocytoma/lipidized medulloblastoma. J Neurooncol 71:53-59, 2005

3. Akhaddar A, Zrara I, Gazzaz M, El Moustarchid B, Benomar S, Boucetta M: Cerebellar liponeurocytoma (lipomatous medulloblastoma). J Neuroradiol 30:121-126, 2003 
4. Alleyne CH Jr, Hunter S, Olson JJ, Barrow DL: Lipomatous glioneurocytoma of the posterior fossa with divergent differentiation: case report. Neurosurgery 42:639-643, 1998

5. Anghileri E, Eoli M, Paterra R, Ferroli P, Pollo B, Cuccarini $\mathrm{V}$, et al: FABP4 is a candidate marker of cerebellar liponeurocytomas. J Neurooncol 108:513-519, 2012

6. Bechtel JT, Patton JM, Takei Y: Mixed mesenchymal and neuroectodermal tumor of the cerebellum. Acta Neuropathol 41:261-263, 1978

7. Buccoliero AM, Caldarella A, Bacci S, Gallina P, Taddei A, Di Lorenzo N, et al: Cerebellar liponeurocytoma: morphological, immunohistochemical, and ultrastructural study of a relapsed case. Neuropathology 25:77-83, 2005

8. Chakraborti S, Mahadevan A, Govindan A, Yasha TC, Santosh V, Kovoor JM, et al: Supratentorial and cerebellar liponeurocytomas: report of four cases with review of literature. J Neurooncol 103:121-127, 2011

9. Davis DG, Wilson D, Schmitz M, Markesbery WR: Lipidized medulloblastoma in adults. Hum Pathol 24:990995, 1993

10. Ellison DW, Zygmunt SC, Weller RO: Neurocytoma/lipoma (neurolipocytoma) of the cerebellum. Neuropathol Appl Neurobiol 19:95-98, 1993

11. Giangaspero F, Cenacchi G, Roncaroli F, Rigobello L, Manetto V, Gambacorta M, et al: Medullocytoma (lipidized medulloblastoma). A cerebellar neoplasm of adults with favorable prognosis. Am J Surg Pathol 20:656-664, 1996

12. González-Cámpora R, Weller RO: Lipidized mature neuroectodermal tumour of the cerebellum with myoid differentiation. Neuropathol Appl Neurobiol 24:397-402, 1998

13. Horstmann S, Perry A, Reifenberger G, Giangaspero F, Huang H, Hara A, et al: Genetic and expression profiles of cerebellar liponeurocytomas. Brain Pathol 14:281-289, 2004

14. Jenkinson MD, Bosma JJ, Du Plessis D, Ohgaki H, Kleihues P, Warnke P, et al: Cerebellar liponeurocytoma with an unusually aggressive clinical course: case report. Neurosurgery 53:1425-1428, 2003

15. Karabagli P, Sav A, Pamir N: Does “cerebellar liponeurocytoma" always reflect an expected site? An unusual case with a review of the literature. Folia Neuropathol 52:101-105, 2014

16. Kleihues P, Cavenee W (eds): World Health Organization Classification of Tumours: Pathology and Genetics of Tumours of the Nervous System. Lyon, France: IARC Press, 2000, pp 110-111
17. Kleihues P, Chimelli L, Giangaspero F, Ohgakin H: Cerebellar liponeurocytoma, in Louis DN, Ohgaki H, Wiestler OD, Cavenee WK (eds): WHO Classification of Tumors of the Central Nervous System. Lyon, France: IARC Press, 2007, pp 110-112

18. Oudrhiri MY, Raouzi N, El Kacemi I, El Fatemi N, Gana R, Maaqili MR, et al: Understanding cerebellar liponeurocytomas: case report and literature review. Case Rep Neurol Med 2014:186826, 2014

19. Pelz D, Khezri N, Mainprize T, Phan N, Keith J, Bilbao J, et al: Multifocal cerebellar liponeurocytoma. Can J Neurol Sci 40:870-872, 2013

20. Radke J, Gehlhaar C, Lenze D, Capper D, Bock A, Heppner FL, et al: The evolution of the anaplastic cerebellar liponeurocytoma: case report and review of the literature. Clin Neuropathol 34:19-25, 2015

21. Taddei GL, Buccoliero AM, Caldarella A, Conti R, Di Lorenzo N, Cacciola F, et al: Cerebellar liponeurocytoma: immunohistochemical and ultrastructural study of a case. Ultrastruct Pathol 25:59-63, 2001

22. Turkalp Z, Karamchandani J, Das S: IDH mutation in glioma: new insights and promises for the future. JAMA Neurol 71:1319-1325, 2014

\section{Disclosures}

The authors report no conflict of interest concerning the materials or methods used in this study or the findings specified in this paper.

\section{Author Contributions}

Conception and design: Hebb, Wolf. Acquisition of data: Hebb, Wolf, Alghefari, Macdonald, Ramsay. Analysis and interpretation of data: Hebb, Wolf, Alghefari, Macdonald, Goobie, Ramsay. Drafting the article: Hebb, Wolf, Alghefari, Krivosheya, Staudt, Bowden, Macdonald, Ramsay. Critically revising the article: Hebb, Wolf, Macdonald, Goobie, Ramsay. Reviewed submitted version of manuscript: Hebb, Wolf, Alghefari, Krivosheya, Staudt, Bowden, Macdonald, Ramsay. Study supervision: Hebb.

\section{Correspondence}

Matthew O. Hebb, Department of Clinical Neurological Sciences, Western University, 339 Windermere Rd., London, Ontario N6A 5A5, Canada.email:mhebb@uwo.ca. 